\title{
Agreement between two diagnostic methods for COVID-19: preliminary data from a Brazilian clinical laboratory
}

\author{
Joyce Regina Santos Raimundo ${ }^{1 *}$, Glaucia Luciano Veiga ${ }^{1}$, Beatriz Costa Aguiar \\ Alves $^{1}$, Jéssica Freitas Araújo Encinas ${ }^{1}$, Marina Cristina Peres ${ }^{1}$, Matheus Moreira \\ Perez ${ }^{1}$, Claudia Giorgia Bronzatti de Oliveira Rodrigues ${ }^{1}$, Aleksandra Vanessa \\ Lambiasi Sant'Anna ${ }^{1}$, Flavia Gehrke ${ }^{1,2}$, Fernando Luiz Affonso Fonseca ${ }^{1,3}$
}

\section{Laboratório de Análises Clínicas, Faculdade de Medicina do ABC, Brazil}

2. Laboratório, Instituto de Assistência Médica ao Servidor Público Estadual de S. Paulo, Brazil

3. Departamento de Ciências Farmacêuticas, Universidade Federal de São Paulo/UNIFESP, Brazil

\begin{abstract}
Objective: To investigate possible differences between laboratory profiles of symptomatic and asymptomatic patients. There are different of them available for COVID-19 diagnoses and surveillance, so this research was to evaluate the positive agreement the diagnostic methods. Methods: For symptomatic patients swab samples from nasal and oral mucosal were collected between first and second week after symptoms onset, to perform RT-PCR, blood samples were collected 7 days after to perform antibody detection test. For asymptomatic patients, only antibody detection was performed to confirm the infection. We investigated specific humoral immune response for symptomatic and asymptomatic patients and also analyzed the positivity index and kappa agreement between immunochromatographic and ELISA assays. Results: Most symptomatic patients presented negative RT-PCR with IgM and IgA detection. Symptomatic and asymptomatic patients have presented elevated IgM and IgA immunoglobulins, being this detection higher in symptomatic patients. The positivity index for immunochromatographic was higher than ELISA and there was no kappa agreement between IgM and IgA detection between these two methods. Conclusion: Symptomatic patients presented higher amounts of IgM and IgA than asymptomatic, suggesting a relation between antibody quantity and severity of disease. We verified no agreement between IgM and IgA detection, and observed higher positivity index for IMMUNO when compared to ELISA. The different kinetics may cause a variation in their detection. Also, many different virus proteins can be used as antigens in these methods, being able of altering their sensibility and specificity.
\end{abstract}

Keywords: COVID-19, symptomatic, diagnostic, asymptomatic

Received: $3^{\text {rd }}$ June 2021; Accepted: $6^{\text {th }}$ September 2021; Published: $19^{\text {th }}$ September 2021

\footnotetext{
* Corresponding author: Joyce Regina Santos Raimundo, Laboratório de Análises Clínicas, Faculdade de Medicina do ABC, Av Lauro Gomes, 2000,09615-085, Santo Andre, Brazil. E-mail: joyce.regina@gmail.com
} 


\section{Introduction}

At the end of 2019, coronavirus SARS-CoV-2 was identified and rapidly spread through all countries generating a world pandemic. The disease known as COVID-19 can cause mild symptoms such as fever and dry cough. In some cases, however, patients can present more severe symptoms such as pneumonia causing serious respiratory complications and organ damage (1). Taking into consideration the high virulence of SARS-CoV-2 and its escalation potential, early diagnosis high sensitivity methods are required to improve prognosis and limit virus spread (2). There are many different serological assays available on the market. They are important diagnostic methods for SARS-CoV-2 to elucidate the patient's immunological response and seroconversion as well as to estimate the disease course for epidemiological studies (3). Serological assays are also important for diagnosis in later stages of the disease, considering the drop in viral load and consequent decrease in sensitivity of RT-PCR and also for diagnostic of asymptomatic patients (4).

Most diagnostic methods focus on IgM detection which proves an acute infection and can be detected between 1 and 2 weeks after first symptoms appear (4). In later stages, it is possible to detect immunoglobulin IgG which provides immune memory. Antibody assays use serological IgA, an acute immunoglobulin, and its serum concentration. Some studies showed that those concentrations rose earlier within 2 days of symptoms. Consequently, the detection of this immunoglobulin could promote an earlier diagnosis for COVID-19 disease $(5,6)$. In human and animal models IgA response against influenza has already been shown to be more effective than induced systemic immunity. Considering this, it is possible that patients with early IgA response for SARS-CoV-2, acquire immunization without disease development (asymptomatic) either with mild or worse symptoms (symptomatic) (7).
The amount of these serological immunoglobulins seems to have a relation with COVID-19 severity. In one study, patients with mild symptoms presented lower IgM detection when compared to severe patients (8), therefore, this dynamics could alter the detection of immunoglobulins in patients with mild or asymptomatic courses of infection (9). Asymptomatic patients, for example, could have lower or undetectable IgA and IgM antibodies in the initial phases of the disease, and only present positive IgG results in later stages. This might impair the early diagnosis and facilitate virus transmission, despite the knowledge that asymptomatic patients have lower chances of transmitting due to the absence of cough and sneezing. However, mild symptomatic patients can also present $\operatorname{IgA}$ and $\operatorname{IgM}$ antibodies that are too low for detection and this may represent a risk because they are more likely to transmit the virus (10).

Considering that early diagnostic and continuous surveillance are important to control SARS-CoV-2 spread, the aim of this study was to investigate an antibody qualitative profile for symptomatic and asymptomatic patients and analyze the positivity index and setting agreement between immunochromatographic (IMMUNO) for IgM and IgG detection, and ELISA assay for IgA and IgG detection, in a preliminary study.

\section{Material and methods}

\section{Blood and oropharyngeal swab samples}

This is a translational study using samples from 125 patients suspected of having COVID-19. They were divided into two main groups: symptomatic, who were experiencing symptoms related to COVD-19, and the asymptomatic group, patients who did not have symptoms of infection, but had contact with diagnosed patients. Of these 125 patients, only 58 patients tested positive for COVID-19, positive diagnostic was considered RT-PCR or/ and antibody detection (IgM/IgG or $\operatorname{IgA} / \operatorname{IgG})$. All positives $\operatorname{IgG}$ were also positive for IgM or IgA immunoglobulins. 
Of the 58 positive patients, 10 of them were symptomatic and 48 asymptomatic. The laboratory inclusion criteria for symptomatic patients were positive RT-PCR or antibody detection, patients with symptoms and negative diagnostic were excluded. For asymptomatic patients, the inclusion criteria were antibody detection, since health services did not request RT-PCR for patients without symptoms. Patients with no symptoms with negative $\operatorname{IgM} / \operatorname{IgG}$ or $\operatorname{IgA} / \operatorname{IgG}$ were excluded. Forty-six patients performed both IMMUNO and ELISA tests, according to medical request in health services.

Symptomatic patients were considered the ones with symptoms related to COVID-19 infection (fever, cough, diarrhea or anosmia/ hyposmia). Nasopharyngeal swabs were collected between first and second week after the onset of symptoms, in accordance with CDC guidelines from symptomatic patients to perform RT-PCR and blood samples were collected 7 days after for antibody detection test. For asymptomatic patients, only antibody detection was performed to confirm COVID-19 infection. For patients who tested positive, we analyzed specific humoral immune response profiles and agreement between IMMUNO and ELISA diagnostic methods.

All patients are from São Paulo and metropolitan regions and were attended in municipal hospitals and clinics. Their samples were processed in the Laboratório de Análises Clínicas do Centro Universitário ABC - Faculdade de Medicina do ABC (FMABC). The present study has been approved by the Ethics and Research Committee of Centro Universitário ABC - Faculdade de Medicina do ABC (FMABC) (protocol 4.067.190).

\section{Molecular test}

RNA extraction from samples was performed using QIAamp Viral RNA Mini Kit (Qiagen, cat no.61904 - Hilden, Germany) according to the manufacturer's instructions.

For RT-PCR tests, fragments of three conserved RNA viral regions (N, RdRP, E) were used for SARS-CoV-2 diagnostic, using SuperScript III
Platinum One-Step qRT-PCR Kit (Invitrogen, cat no. 11732020 - EUA) kit, following manufacturer's protocol. Twenty microliters of reaction mix consisting of $2 \times$ RT-PCR buffer, enzyme mix, primers-probes, and $5 \mu$ of extracted nucleic acid. RT-PCR was performed on an ABI 7500 real-time PCR system (Applied Biosyste$\mathrm{ms}$ ) with the following cycle parameters: $15 \mathrm{~min}$ at $50^{\circ} \mathrm{C}$ for reverse transcription, and $2 \mathrm{~min}$ of inactivation at $95^{\circ} \mathrm{C}$ followed by 40 cycles of $15 \mathrm{~s}$ at $95^{\circ} \mathrm{C}$, and $30 \mathrm{~s}$ at $60^{\circ} \mathrm{C}$.

\section{Immunoassay (Rapid Test)}

For $\operatorname{IgM}$ and $\operatorname{IgG}$ detection, blood samples obtained by venipuncture were used and Instant-view COVID-19 IgG/IgM AlfaTest (Poway, CA 92064 - USA) kit was used, following the manufacturer's instructions. Using IMMUNO method, the test identifies the binding of antibodies from $\operatorname{IgM}$ and $\mathrm{IgG}$ classes present in the blood sample with a specific SARS-CoV-2 antigen fixed in a nitrocellulose tape, and the addition of an enzymatic substrate generates a coloring when antigen-antibody binding occurs. The test provides qualitative results, indicating presence or absence of antibodies. Patients' results that presented color line on IgM and/or IgG demarcation were consider positive. The manufacturer's manual informs sensitivity of $99 \%$ and specificity of $94 \%$, however, no information of viral proteins used as antigen were elucidate. The samples were stored under refrigeration $\left(5-8^{\circ} \mathrm{C}\right)$.

\section{ELISA}

Blood samples to perform the test were obtained by venipuncture and plasma was used for antibody detection. The samples were stored under refrigeration $\left(5-8^{\circ} \mathrm{C}\right)$. For IgA detection the Euroimmun Anti-SARS-CoV-2 ELISA IgA (Lubeck - German) kit was used following the manufacturer's instructions. For IgG detection Euroimmun Anti-SARS-CoV-2 ELISA IgG (Lubeck - German) kit was used following the manufacturer's instructions. Using ELISA method, the 
test identifies the binding of antibodies from $\operatorname{Ig} \mathrm{A}$ or IgG classes present in blood samples using as antigen fragments of domain S1 of the SARSCoV-2 spike protein. The colorimetric reaction provides semiquantitative results by a ratio calculated by the device, patients with ELISA ratio $\geq 1.1$ were considered positive. The manufacturer's manual informs sensitivity of $90 \%$ and specificity of $98 \%$.

\section{Statistical Analysis}

The data were expressed in absolute and relative values. Kappa test measures the degree of agreement between ratios derived from sample dependents. For this, positive and negative results from IMMUNO were compared with positive and negative results from ELISA by GraphPad Prism 6, with a 95\% confidence level. Determination of agreement were analyzed by kappa index, provided by the software after analysis: kappa < 0: no agreement; kappa between 0.00 and 0.20: slight agreement; kappa between 0.21 and 0.40: fair agreement; kappa between 0.41 and 0.60: moderate agreement; kappa between 0.61 and 0.80: substantial agreement; kappa between 0.81 and 1.00: almost perfect agreement. Laboratory profile in symptomatic patients, acute immunoglobulin profile in asymptomatic and symptomatic patients, and positivity index between IMMUNO and ELISA were estimated by percentage, as described in the results section.

\section{Results}

In this study, samples from 125 patients were tested for COVID-19. Of the 58 positive patients, $8 \%$ were coursing symptomatic infection and $38 \%$ were asymptomatic. Sample characteristics are described in table 1.

Samples from symptomatic patients were analyzed by RT-PCR as well as by antibody detection by IMMUNO (IgM and IgG) and ELISA (IgA and $\mathrm{IgG}$ ) methods. Ten patients who tested positive were considered $100 \%, 9$ of them presented positive IgM and negative RT-PCR, 6 presented positive IgA and negative RT-PCR, and 2 patients had positive IgA with positive RTPCR (table 2).

Samples from asymptomatic patients were analyzed only by IMMUNO and ELISA assays. For acute immunoglobulin profile in asymptomatic ones, 48 patients who tested positive were considered as $100 \%, 39$ patients tested positive for IgM, and 27 tested positive for IgA. For symptomatic ones, 10 patients who tested positive were considered as $100 \%, 9$ patients tested positive for $\operatorname{IgM}$, and 8 tested positive for IgA (Table 3 ).

To determine the agreement between IMMUNO and ELISA, we performed kappa index between $\operatorname{IgM}$ (IMMUNO) and IgA (ELISA), and between IgG (IMMUNO) and IgG (ELISA) (table 4).

Table 1. Sample caracterization.

\begin{tabular}{lcc}
\hline Characteristics & $\mathbf{N}$ & $\mathbf{\%}$ \\
\hline Total patients & 125 & - \\
\hline RT-PCR & 13 & 10 \\
\hline IMMUNO IgM/IgG & 79 & 63 \\
\hline ELISA IgA/IgG & 92 & 73 \\
\hline Positives & 58 & 46 \\
\hline Negatives & 67 & 53 \\
\hline Positive symptomatics & 10 & 8 \\
\hline Positive asymptomatics & 48 & 38 \\
\hline Sex & & \\
Female & 87 & 70 \\
Male & 38 & 30 \\
\hline Age & & \\
Median & Minimum & Maximum \\
39 & 19 & 78 \\
\hline
\end{tabular}

Table 2. Laboratory profile for symptomatic patients with positive diagnostic.

\begin{tabular}{ll}
\hline Positive symptomatic & N (\%) \\
\hline IgM + and RT-PCR - & $9(90)$ \\
\hline IgA + and RT-PCR - & $6(60)$ \\
\hline IgA + and RT-PCR + & $2(20)$ \\
\hline
\end{tabular}

Note: + and - symbols means positive and negative, respectively.

Table 3. Acute immunoglobulin profile for asymptomatic and symptomatic patients with positive diagnostic.

\begin{tabular}{lcc}
\hline & $\begin{array}{c}\text { Positive } \\
\text { asymptomatic } \\
\text { N (\%) }\end{array}$ & $\begin{array}{c}\text { Positive } \\
\text { symptomatic } \\
\mathbf{N}(\mathbf{\%})\end{array}$ \\
\hline IMMUNO IgM & $39(81)$ & $9(90)$ \\
\hline ELISA IgA & $27(56)$ & $8(80)$ \\
\hline
\end{tabular}


Table 4. agreement between IgM (IMMUNO) and IgA (ELISA) and agreement between IgG (IMMUNO) and IgG (ELISA) (kappa index)

\begin{tabular}{|c|c|c|c|c|c|}
\hline \multirow{5}{*}{ ELISA (IgA) } & \multicolumn{4}{|c|}{ IMMUNO (IgM) } & \\
\hline & & Positive & Negative & Total & \multirow{4}{*}{$\begin{array}{c}\text { Kappa }=-0.131 \\
\text { Kappa }<0: \text { No agreement }\end{array}$} \\
\hline & Positive & 23 & 6 & 29 & \\
\hline & Negative & 22 & 2 & 24 & \\
\hline & Total & 45 & 8 & 53 & \\
\hline \multirow{5}{*}{ ELISA (IgG) } & \multicolumn{4}{|c|}{ IMMUNO (IgG) } & \\
\hline & & Positive & Negative & Total & \\
\hline & Positive & 11 & 2 & 13 & Kappa $=0.559$ \\
\hline & Negative & 8 & 32 & 40 & Kappa between 0.41 and \\
\hline & Total & 19 & 34 & 53 & 0.60 : moderate agreement \\
\hline
\end{tabular}

To verify the total positivity index between IMMUNO and ELISA tests we analyzed the percentage of positive diagnoses between these two methods. For IMMUNO, 58 positive patients were considered as $100 \%, 45$ patients with positive IgM detection, and 19 patients with positive IgG. For ELISA, 58 positive patients were considered as 100\%, 29 patients with positive IgA detection, and 13 patients with positive IgG (table 5).

Table 5. Positivity index between IMMUNO and ELISA methods.

\begin{tabular}{lcc}
\hline & N & \% \\
\hline IMMUNO & & \\
IgM & 45 & 77 \\
IgG & 19 & 33 \\
\hline ELISA & & \\
IgA & 29 & 50 \\
IgG & 13 & 22 \\
\hline
\end{tabular}

\section{Discussion}

In this study, we aimed to investigate a qualitative laboratory profile for COVID-19 symptomatic and asymptomatic patients, and, also to evaluate the agreement and positivity index between IMMUNO and ELISA diagnostic tests. In the studied population, most symptomatic patients (90\%) presented $\operatorname{IgM}$ antibodies and negative RT-PCR, 60\% presented positive IgA and negative RT-PCR, and only $20 \%$ presented positive
IgA and positive RT-PCR. These results could be due to a late sample collecting time when the viral load was already in decline, decreasing viral load concentration (11). Most of the positive patients in this study presented immunoglobulin detection of $\operatorname{IgM}$ and IgA, which are considered acute phase antibodies (12), with IgM detection being most prevalent (13).

For asymptomatic patients considered positive by serological assays, positivity rate of RT-PCR is low - around 30\%; considering this, in our study, asymptomatic patients did not perform RT-PCR. This low detection seems to occur because viral load is linked to symptom intensity (14). This leads to a discussion about asymptomatic patients' transmissibility, because of the absence of cough and sneezing (10). It was already shown that norovirus transmissibility coefficients for asymptomatic patients were low, so they had minimal chances of spreading the virus (15). Concerning COVID-19, studies have shown that viral nucleic acids can be detected in infected people one to two days before the onset of symptoms, so even patients who present asymptomatic infection could be transmitters during the course of infection, but as the viral load is low, they are less likely to transmit (16). Confirming this, a study evaluated the transmission in symptomatic and asymptomatic infections and observed that symptomatic patients are able to transmit the virus for up to 3 peo- 
ple while asymptomatic patients transmit to less than one. This means that in asymptomatic cases the chances of transmission to only one person is lower. (17).

Regarding the acute immunoglobulin profiles for asymptomatic and symptomatic patients, we observed that asymptomatic ones presented IgM and IgA detection, with IgM detection being more prevalent than $\operatorname{IgA}(81 \%$ vs. $56 \%)$. Symptomatic patients also presented higher IgM and IgA detection ( $90 \%$ vs. $80 \%$ ) in comparison with asymptomatic patients. These observations corroborate with studies correlating immunoglobulin amount with COVID-19 severity (8, 9). About positivity agreement, the kappa index showed no agreement between IgM detection by IMMUNO and IgA detection by ELISA (kappa -0.131). The different kinetics between these two acute immunoglobulins and different viral proteins used as antigens by these assays might have an influence in their sensitivity altering their correlation. A study observed that sensitivity for IgA detection was higher in the first week of symptoms and IgM sensitivity increased after one week of the onset of symptoms, so depending on the time of collection it is possible that only IgA or IgM is detectable (18). For IgG detection between both methods, the kappa index showed moderate agreement, minor differences were observed that might be also related to different viral proteins used by both assays (19). In our study, we observed that the positivity index was higher for IMMUNO than ELISA assay (89\% for IgM and 32 for IgG vs. 63 for IgA and $27 \%$ for IgG, respectively). Many COVID-19 diagnostic assays of different brands are available and most of them do not inform which viral proteins are used in their manufacture and this information could be important to better understand their specificity. SARS-CoV-2 S and SARS-CoV S protein, for example, are very similar, sharing $75 \%$ of amino acid sequence (20). A study showed that SARS-CoV S protein promotes polyclonal antibody responses which neutralized SARS-CoV-2 S protein-mediated entry into cells. Therefore, it might be difficult to identify specific immunoglobulins in serological studies and cross reactions may occur (20). Using all subunits of $\mathrm{S}$ protein as antigens also decreases the specificity, thus, possibly generating cross-reactivity (21), therefore, S1 domain appears to be more specific for SARS-CoV-2. These sensitivity differences between test compositions might impair asymptomatic diagnosis because there is no clinical-laboratory correlation. The diagnosis is only based on test results, so considering the variability of sensitivity; the question is whether some of these results could be false positive (22).

Other coronaviruses also share the RBD in $\mathrm{S}$ protein with SAR-CoV-2, but despite their similar structural homology, many residues differ between these two RBDs (23). A study demonstrated that antibodies for SARS-CoV RBD did not bind to SAR-CoV-2 RBD (24), so using this region as antigen, might also provide more specific diagnostic methods (22). The most abundant protein in SAR-CoV-2 is the nucleocapside (N) protein and plenty of antigens are also produced against it, but this protein is common in other viruses, so a cross reaction can also occur $(2,11)$. Taking into consideration the differences in specificity of all the discussed proteins for SARCoV-2, diagnostic methods that use S1 or RBD proteins as antigens might be more specific and sensitive in the diagnosis of COVID-19. The combination of more than one specific protein might increase sensitivity of detection of assays (21). Thus, different diagnostic methods can present variable detection sensitivities and depending on which proteins one uses as an antigen target, false positive results may occur.

\section{Consclusion}

In this study, symptomatic patients presented higher detection of IgM and IgA than asymp- 
tomatic patients, corroborating with studies showing that antibody amounts are related to the severity of the disease. We verified no agreement between IgM measured by IMMUNO and IgA measured by ELISA, and observed higher positivity index for IMMUNO when compared to ELISA. Despite the fact that both immunoglobulins are considered typical for the acute phase, the differences in their kinetics may cause a variation in their detection. Also, many different virus proteins can be used as antigens in these methods, being able of altering their sensibility and specificity. The use of less specific proteins could lead to false positive results as well.

\section{Authors' contribution}

All authors contributed equally to the production of this work.

\section{Conflicts of interest}

None to declare.

\section{References}

1. Ge H, Wang X, Yuan X, Xiao G, Wang C, Deng T, et al. The epidemiology and clinical information about COVID-19. Eur J Clin Microbiol Infect Dis. 2020;39(6):1011-9. DOI: 10.1007/s10096-020-03874-z

2. Sethuraman N, Jeremiah SS, Ryo A. Interpreting Diagnostic Tests for SARS-CoV-2. JAMA. 2020 Jun 9;323(22):2249-51. DOI: 10.1001/jama.2020.8259

3. Padoan A, Sciacovelli L, Basso D, Negrini D, Zuin S, Cosma $\mathrm{C}$, et al. IgA-Ab response to spike glycoprotein of SARS-CoV-2 in patients with COVID-19: A longitudinal study. Clin Chim Acta. 2020;507:164-6. DOI: 10.1016/j.cca.2020.04.026

4. Zhao J, Yuan Q, Wang H, Liu W, Liao X, Su Y, et al. Antibody responses to SARS-CoV-2 in patients of novel coronavirus disease 2019. Clin Infect Dis. 2020 Nov 19;71(16):2027-34. DOI: 10.1093/cid/ciaa344

5. Chao YX, Rötzschke O, Tan EK. The role of IgA in COVID-19. Brain Behav Immun. 2020;87:182-3. DOI: 10.1016/j.bbi.2020.05.057

6. Yu HQ, Sun BQ, Fang ZF, Zhao JC, Liu XY, Li YM, et al. Distinct features of SARS-CoV-2-specific IgA response in COVID-19 patients. Eur Respir J. 2020 Aug; 56(2): 2001526. DOI: 10.1183/13993003.01526-2020
7. Azkur AK, Akdis M, Azkur D, Sokolowska M, van de Veen W, Brüggen MC, et al. Immune response to SARS-CoV-2 and mechanisms of immunopathological changes in COVID-19. Allergy. 2020 Jul;75(7):156481. DOI: $10.1111 /$ all.14364

8. Wang Y, Zhang L, Sang L, Ye F, Ruan S, Zhong B, et al. Kinetics of viral load and antibody response in relation to COVID-19 severity. J Clin Invest. 2020 Oct 1;130(10):5235-44. DOI: 10.1172/JCI138759

9. Traugott M, Aberle SW, Aberle JH, Griebler H, Karolyi M, Pawelka E, et al. Performance of Severe Acute Respiratory Syndrome Coronavirus 2 Antibody Assays in Different Stages of Infection: Comparison of Commercial Enzyme-Linked Immunosorbent Assays and Rapid Tests. J Infect Dis. 2020;222(3):362-6. DOI: 10.1093/ infdis/jiaa305

10. Gao M, Yang L, Chen X, Deng Y, Yang S, Xu H, et al. A study on infectivity of asymptomatic SARS-CoV-2 carriers. Respir Med. 2020;169:106026. DOI: 10.1016/j. rmed.2020.106026

11. To KK, Tsang OT, Leung WS, Tam AR, Wu TC, Lung $\mathrm{DC}$, et al. Temporal profiles of viral load in posterior oropharyngeal saliva samples and serum antibody responses during infection by SARS-CoV-2: an observational cohort study. Lancet Infect Dis. 2020;20(5):56574. DOI: 10.1016/S1473-3099(20)30196-1

12. Guo L, Ren L, Yang S, Xiao M, Chang, Yang F, et al. Profiling Early Humoral Response to Diagnose Novel Coronavirus Disease (COVID-19). Clin Infect Dis. 2020. DOI: $10.1093 / \mathrm{cid} / \mathrm{ciaa} 310$

13. Paces J, Strizova Z, Smrz D, Cerny J. COVID-19 and the immune system. Physiol Res. 2020 Jul 16;69(3):37988. DOI: $10.33549 /$ physiolres.934492

14. Meng H, Xiong R, He R, Lin W, Hao B, Zhang L, et al. $\mathrm{CT}$ imaging and clinical course of asymptomatic cases with COVID-19 pneumonia at admission in Wuhan, China. J Infect. 2020;81(1):e33-e9. DOI: 10.1016/j. jinf.2020.04.004

15. Chen T, Gu H, Leung RK, Liu R, Chen Q, Wu Y, et al. Evidence-Based interventions of Norovirus outbreaks in China. BMC Public Health. 2016;16(1):1072. DOI: 10.1186/s12889-016-3716-3

16. $\mathrm{Wu} \mathrm{ZY}$. [Contribution of asymptomatic and pre-symptomatic cases of COVID-19 in spreading virus and targeted control strategies]. Zhonghua Liu Xing Bing Xue Za Zhi. 2020;41(6):801-5.

17. Chen Y, Wang AH, Yi B, Ding KQ, Wang HB, Wang $\mathrm{JM}$, et al. [Epidemiological characteristics of infection in COVID-19 close contacts in Ningbo city]. Zhonghua Liu Xing Bing Xue Za Zhi. 2020;41(5):667-71.

18. Montesinos I, Gruson D, Kabamba B, Dahma H, Van den Wijngaert S, Reza S, et al. Evaluation of two automated and three rapid lateral flow immunoassays for the detection of anti-SARS-CoV-2 antibodies. J Clin Virol. 2020;128:104413. DOI: 10.1016/j.jcv.2020.104413 
19. Lisboa Bastos M, Tavaziva G, Abidi SK, Campbell JR, Haraoui LP, Johnston JC, et al. Diagnostic accuracy of serological tests for covid-19: systematic review and meta-analysis. BMJ. 2020;370:m2516. DOI: 10.1136/ bmj.m 2516

20. Walls AC, Park YJ, Tortorici MA, Wall A, McGuire AT, Veesler D. Structure, Function, and Antigenicity of the SARS-CoV-2 Spike Glycoprotein. Cell. 2020;181(2):281-92.e6. DOI: 10.1016/j. cell.2020.02.058

21. Okba NMA, Müller MA, Li W, Wang C, GeurtsvanKessel CH, Corman VM, et al. Severe Acute Respiratory Syndrome Coronavirus 2-Specific Antibody Responses in Coronavirus Disease Patients. Emerg Infect Dis. 2020;26(7):1478-88. DOI: 10.3201/eid2607.200841

22. Ou X, Liu Y, Lei X, Li P, Mi D, Ren L, et al. Character- ization of spike glycoprotein of SARS-CoV-2 on virus entry and its immune cross-reactivity with SARS-CoV. Nat Commun. 2020;11(1):1620. DOI: 10.1038/s41467020-15562-9

23. Othman H, Bouslama Z, Brandenburg JT, da Rocha J, Hamdi Y, Ghedira K, et al. Interaction of the spike protein RBD from SARS-CoV-2 with ACE2: Similarity with SARS-CoV, hot-spot analysis and effect of the receptor polymorphism. Biochem Biophys Res Commun. 2020 Jun 30;527(3):702-8. DOI: 10.1016/j. bbrc.2020.05.028

24. Wrapp D, Wang N, Corbett KS, Goldsmith JA, Hsieh CL, Abiona O, et al. Cryo-EM structure of the 2019$\mathrm{nCoV}$ spike in the prefusion conformation. Science. 2020;367(6483):1260-3. DOI: 10.1126/science. abb2507 